\title{
Harmonic Generation in $\mathrm{N}_{\mathbf{2}}$ and Ar by Wave-Mixing
}

\author{
F. Giammanco, P. Ceccherini, C. Tagliavini, M. Malvezzi*; P. Villoresi** and \\ G. Tondello**
}

Dipartimento di Fisica, Piazza Torricelli, 2-56126 Pisa, Italy

* Dipartimento di Elettronica, via Ferrata, 1-27100 Pavia, Italy

** Dipartimento di Elettronica e Informatica, via Gradenigo, 6/a-35128 Padova, Italy

\begin{abstract}
Harmonic generation in gases has been investigated by mixing of fundamental $(1064 \mathrm{~nm})$ and second harmonic $(532 \mathrm{~nm})$ of a $100-\mathrm{mJ}$ 35-ps Nd:YAG laser. Even and odd harmonics of fundamental wavelength are generated by wave-mixing. We have investigated in detail harmonic spectrum in $\mathrm{Ar}$ and $N_{2}$, up to the $14^{\text {th }}$ harmonics, versus the gas pressure and laser intensity.
\end{abstract}

\section{INTRODUCTION}

The phenomenon of the harmonic generation in gaseous media has been studied extensively by many research groups as an alternative method of producing shortwavelength coherent radiation [1]. Harmonic spectrum generated in noble gases by a 30-ps Nd:YAG laser with a peak power of $5 \times 10^{13} \mathrm{~W} / \mathrm{cm}^{2}$ exhibits a plateau and ends up with a sharp cut-off [2]. Plateau extension increases as the laser wavelength increases according to a semi-empirical relation $E_{\max } \approx I_{p}+3.2 U_{p}$ (where $E_{\max }$ is the maximum harmonic energy, $I_{p}$ is the ionization potential and $U_{p}$ its ponderomotive shift). Attention has been mainly focused on harmonic generation in rare gases by single-frequency excitation. We have analyzed the harmonic spectrum in Ar and $\mathrm{N}_{2}$ by wave-mixing of parallel polarized fundamental $(1064 \mathrm{~nm})$ and second harmonics $(532 \mathrm{~nm})$ of a picosecond Nd:YAG laser in the region from the $3^{\text {rd }}$ to $14^{\text {th }}$ of fundamental, as a function of gas density and laser intensity. The experimental setup is quite common to this kind of experiments. The laser beam is fed into a vacuum chamber which contains a pulsed valve to generate the gas jet, and harmonics are detected along the laser direction by a monocromator in the Seya-Namioka configuration. We have performed a calibration of detection components. More detailed information about experimental setup and calibration are described in Ref. [3].

\section{EXPERIMENTAL RESULTS}

Shapes of harmonic spectra, recorded in Ar and $N_{2}$, are quite similar, [figs 1 (a) and (b)]. Hence, the structure of bound levels does not affect harmonic generation. Since ionization potential of $\mathrm{Ar}$ and $N_{2}$ are quite close, the spectra likeness can be understood from the point of view of the plasma model [3] [4]. In addition, we have detected harmonic spectra when the chamber is uniformelly filled by gas. In this case, the detectable spectrum is limited by the absorption of gas background. We observed a strong gain of some harmonics as compared with the ones detected in the gas jet. Conversion efficiency enhancement in the uniform density mode can be explained in terms of an increase of the active length and hence as a sort of spatial amplification along the interaction region. Spectra recorded versus pressure, in both configuration, do not show evidence of Phase-Matching modulation, [fig 2 (a)]. This can be explained 
by collective effects in the MPI plasma [4]. At density below $10^{18} \mathrm{at}(\mathrm{mol}) / \mathrm{cm}^{3}$, accordingly with plasma model, the intensity of q-th harmonic has a cubic dependence on gas density. Data recorded versus laser intensity are not in agreement with the weak-field approximation. Some discrepancies arise especially for $I_{1 \omega}<2 \times 10^{13} \mathrm{~W} / \mathrm{cm}^{2}$ for harmonics order higher than the $6^{\text {th }}$ in Ar. To account this behavior and to test applicability of plasma model, we use a simplified version of model under weak-field approximation, including the ionization of the medium, [fig. 2 (b)].

Let us remark that brilliance of source realized in this experiment $\left(2 \times 10^{16}-2 \times\right.$ $10^{18} \frac{\text { photons }}{s \cdot \mathrm{cm}^{2} \cdot \text { sterad }}$ ) overcomes by at least one order of magnitude that the one measured in commercial sources.
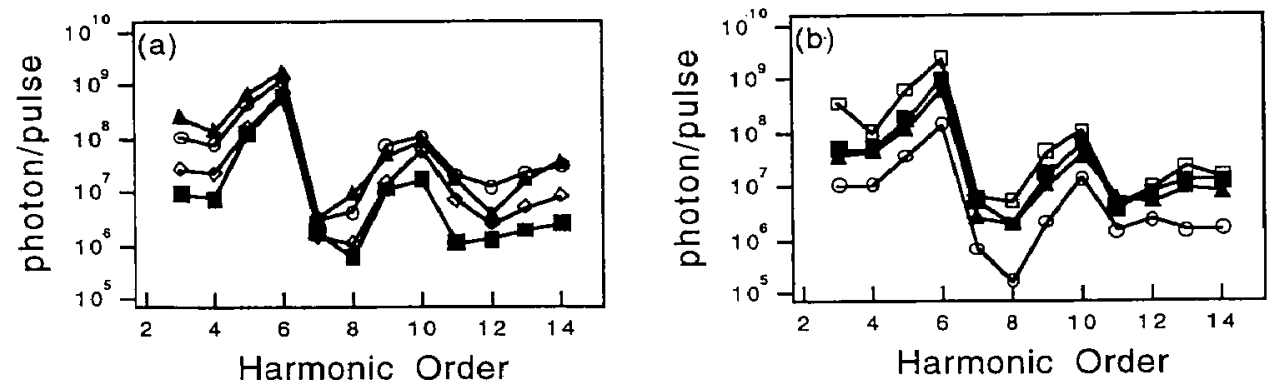

Figure 1: (a) Photon per pulse in Ar at gas density $1.2 \times 10^{18}$ (Full squares), $2.1 \times 10^{18}$ (Open diamonds), $4 \times 10^{18}$ (Open circles) and $6 \times 10^{18}$ (Full triangles) atoms $/ \mathrm{cm}^{3}$. (b) Photon per pulse in $N_{2}$ at gas density $2.5 \times 10^{17}$ (Open circles), $5.5 \times 10^{17}$ (Full triangles), $1 \times 10^{18}$ (Full squares) and $2 \times 10^{18}$ (Open squares) molecules $/ \mathrm{cm}^{3}$.
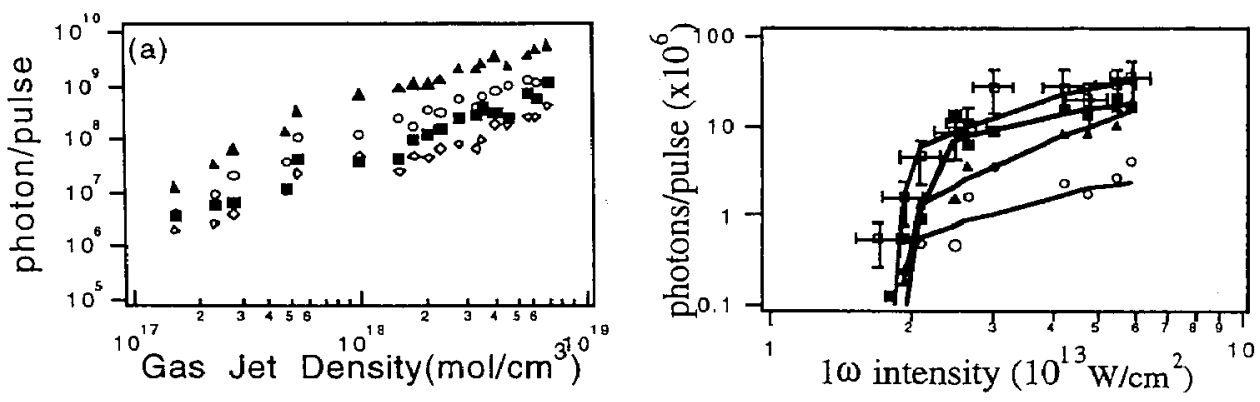

Figure 2: (a) Photons per pulse vs $N_{2}$ gas jet density. Full squares-3 $3^{\text {rd }}$, Open diamonds-4 $4^{t h}$, Open circles-5 $5^{\text {th }}$ and Full triangles- $6^{\text {th }}$. (b) Ar-gas jet density $6 \times 10^{18}$ atoms $/ \mathrm{cm}^{3}$-wave-mixing. Full squares-1 $11^{\text {th }}$, Open circles-12 $2^{\text {th }}$, Full triangles-1 $3^{\text {th }}$ and Open squares-1 $14^{\text {th }}$. Continous lines-best fit.

\section{References}

[1] Reintjes J.F., Nonlinear Optical Parametric Processes in Liquids and Gases (Academic, New York, 1984).

[2] Li X.F. et al., Phys. Rev. A 39 (1989) 5751.

[3] Giammanco et al., "Harmonic Generation in $\mathrm{N}_{2}$ and Ar by Wave-Mixing", F. Giammanco and N. Spinelli, Eds., Plasma Collective Effects in Atomics Physics (Edizioni ETS, Pisa, 1996).

[4] Giammanco F., Phys.Rev. A 42 (1991) 6939. 